\title{
Anharmonic coupling between electrons and TO phonons in the vicinity of a ferroelectric quantum critical point
}

\author{
P. Chudzinski 10 \\ ASC, School of Mathematics and Physics, Queen's University Belfast, Belfast BT7 1NN, United Kingdom
}

(Received 11 June 2019; revised manuscript received 30 September 2019; accepted 27 January 2020; published 27 February 2020)

\begin{abstract}
We explore a mechanism that allows to couple electrons with the transverse-optical (TO) phonon branch in a regime when the TO mode becomes highly anharmonic and drives the ferroelectric phase transition. We show that this anharmonicity, which leads to a collective motion of ions, is able to couple electronic and lattice displacement fields. An effective correlated electron-ion dynamics method is required to capture the effect of the onset of the local electric polarization due to this collective behavior close to the quantum critical point. We identify an intermediate temperature range where an emergent phonon drag may contribute substantially to thermoelectric conductivity in this regime. We find that, under optimal conditions, this extra contribution may be larger than values achieved so far in the benchmark material, PbTe. In the last part we make a case for the importance of our results in the generic problem of anharmonic electron-lattice dynamics.
\end{abstract}

DOI: 10.1103/PhysRevResearch.2.012048

Motivated by the search for new efficient renewable sources of energy, a huge experimental effort has been made towards identifying materials with better thermoelectric properties in recent years. So far, this intense search has been based mostly on a single-particle theoretical description of electrons and standard electron-phonon coupling due to displacement potential, supported by numerical ab initio simulations [1,2]. While this research program has had some notable successes [3] it should be also noted that the progress has been slow and so novel pathways are needed. At the same time it has been observed that in several cases good thermoelectrics (TE) are weakly doped semiconductors, such as PbTe [4], $\mathrm{SnTe}$, or $\mathrm{SrTiO}_{3}$, that are in the vicinity of a ferroelectric quantum critical phase transition (FE-QCP). It is then natural to ask if it is just a pure coincidence, or whether a yet undiscovered mechanism enhances the Seebeck coefficient.

As the system approaches the FE-QCP, a crucial role is played by the transverse-optical (TO) phonons. In the Landau macroscopic framework, the TO mode spectral weight is coupled to the material's electric polarization [5] (the order parameter), so as the FE transition takes place, the TO softening at $q=0$ indicates the emergence of a uniform displacement and the polar order. The electrons should be susceptible to this dipole ordering and usually the appearance of the FE is accompanied by a disappearance of the electron pockets. Otherwise the remaining free electrons would screen the FE order. A massive softening at the $\Gamma$ point implies that the TO branch emerges as a new family of phonons with

Published by the American Physical Society under the terms of the Creative Commons Attribution 4.0 International license. Further distribution of this work must maintain attribution to the author(s) and the published article's title, journal citation, and DOI. a very substantial velocity, often comparable to that of the longitudinal-acoustic (LA) branch [6], and so they certainly contribute to the transport properties of the system.

The assertion about coupling between electrons and the TO phonons is experimentally confirmed in an incipient $\mathrm{FE}, \mathrm{PbTe}$, by observation [7] that (i) photoexcited electrons do change the TO phonons frequency and (ii) in return these TO phonons do modify the electrons' spectral gap. However, within the Fermi liquid framework it is assumed that the TO phonons are only very weakly coupled with electrons. This is based on the fact that the vector of polarization of the Fermi liquid and the displacement vector associated with the TO mode are perpendicular to each other [8], which diminishes their coupling (see Fig. 1). Even the most recent $a b$ initio studies dedicated to PbTe $[9,10]$ conventionally claimed that the TO phonons have the weakest coupling with electrons [11]. This has led to the contradictory conclusion that electron coupling with the TO phonons will play a very minor role close to the FE-QCP. Clearly, the TO coupling must emerge at a collective level, which is not captured in a single-particle picture, and there is an obvious demand for theory to uncover the microscopic mechanism. An emergent model of correlated electron-ion dynamics in a nonadiabatic regime is needed to make an unbiased proof of the energy transfer. This is the key result of this work.

The mechanism of electron-phonon coupling, present close to the FE-QCP, can be illustrated by a phenomenological argument (see Fig. 1). An underlying reason for the TO phonon softening is the long-range interactions between lattice vibrations. Such interactions change the character of the ion potential from quadratic to quartic [6] along the line of FE distortion. In second quantization language $\hat{x}^{4}$ translates into phonon-phonon interactions. The TO phonon is not an eigenstate of the lattice oscillations anymore; in its motion it is accompanied by a cloud of other low-energy phonons [12]. 


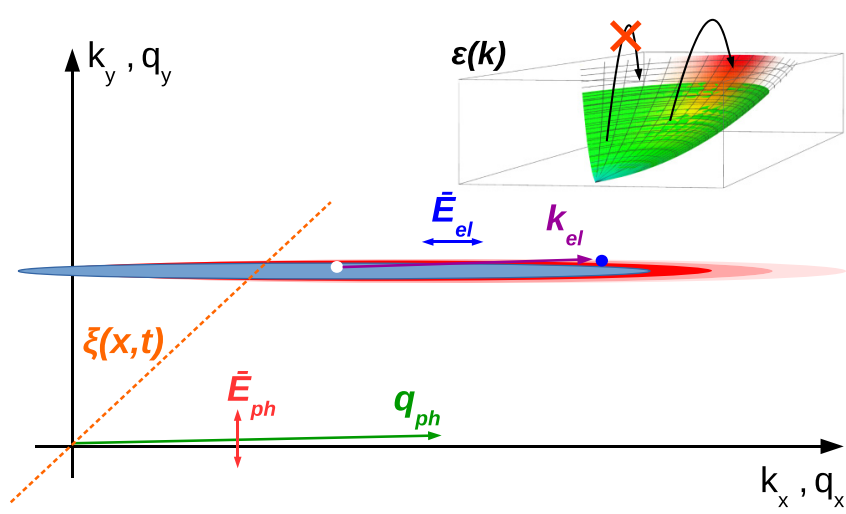

FIG. 1. In the conventional model for coupling between electron and lattice displacements an electron-hole excitation in a Fermi pocket with momentum $k_{e l}$ (blue) creates an electric field $\vec{E}_{e l}$ while the matching TO mode with momentum $q_{p h}$ (green), $q_{p h}=k_{e l}$, moves lattice atoms in a way that induces an electric field $\vec{E}_{p h}$ such that $\vec{E}_{p h} \perp \vec{E}_{e-h} \equiv \vec{E}_{p h} \cdot \vec{E}_{e l}=0$, i.e., negligible coupling. The novel electron-TO phonon coupling mechanism is possible in the presence of an accompanying lattice distortion described by a generalized collective variable $\xi(x, t)$ (with its direction indicated by an orange dashed line). Inset: A slice of Fermi pocket showing a dispersion $\epsilon(k)$ in the vicinity of the heavy $x$ axis. In red we show displacement of electronic density localized around the $x$ axis due to the energymomentum conservation that prohibits electron-phonon scattering processes away from the heaviest electron direction.

Physically this cloud of coexcited phonons (i.e., their displacements $\Xi_{q}$ ) is resummed into a collective variable, a vector field $\vec{\xi}(x, t)$ that describes the ordering of the system in the vicinity of the QCP $[5,13]$. In the FE-QCP case the ordering is equivalent to an electric polarization of the system. Then, as illustrated in Fig. 1, the field $\vec{\xi}(x, t)$ accompanying a TO mode induces an electric displacement field with a component in the direction of the electron liquid polarization. This configuration of induced local electric fields has a nonnegligible energy, hence we have identified the new source of electron-phonon coupling. The strength of the electron-TO phonon coupling is proportional to the symmetry breaking $\vec{\xi}(x, t)$ which we consider as a dynamical variable. The aim of our study is to show that such a nonadiabatic electron-phonon coupling is not only non-negligible, but can give a substantial net effect on the transport properties.

We consider narrow gap, weakly doped semiconductors with extremely small carrier densities whose Hamiltonian is

$$
\begin{aligned}
H= & \sum_{k} V_{F} k c_{k}^{\dagger} c_{k}+\sum_{q} \omega_{\mathrm{TO}}(q) b_{q}^{\dagger} b_{q} \\
& +\sum_{k, q} V_{\mathrm{ee}}(k, q) c_{k 1}^{\dagger} c_{k 2} c_{k 3}^{\dagger} c_{k 4} \\
& \times\left.\delta\left(k_{1}+k_{3}-k_{2}-k_{4}\right)\right|_{k=k_{1}+k_{3}, q=k_{1}-k_{2}} \\
& +\sum_{q, q^{\prime}} U_{\mathrm{ah}}\left(q, q^{\prime}\right) b_{q 1}^{\dagger} b_{q 2} b_{q 3}^{\dagger} b_{q 4} \\
& \times\left.\delta\left(q_{1}+q_{3}-q_{2}-q_{4}\right)\right|_{q=q_{1}+q_{3}, q^{\prime}=q_{1}-q_{2}},
\end{aligned}
$$

where the terms describe electron dispersion ( $c_{k}^{\dagger}$ operators), phonon dispersion ( $b_{q}^{\dagger}$ operators), electron-electron interactions $V_{\mathrm{ee}}(k, q)$, and phonon-phonon interactions $U_{\mathrm{ah}}\left(q, q^{\prime}\right)$. The linear dispersion of fermions with velocity $V_{F}$ is justified in a narrow energy window (of the order of a few terahertz) close to $E_{F}$. We expect the Fermi surface (FS) to be highly anisotropic, since the FSs with a high complexity factor [3] are common in TE, with a large effective mass in at least one direction, and we study the dynamics along the $\hat{x}$ direction with the lowest value of $V_{F}$. The most prominent feature of our system is its proximity to FE-QCP (although on the quantum paraelectric side) and the parameters of the model must reflect this. A sole assumption of a long-range nature of $U_{\text {ah }}$ [14] (needed for FE) suffice [15] to strongly renormalize downwards $\omega_{T O}(q \rightarrow 0)$ and induce a large self-energy experimentally detected as broadening-the waterfall effect [16]. For larger $q$ the TO phonons become strongly dispersive and contribute substantially to transport. This is anomalous for this phonon branch and puts it beyond the adiabatic regime. A microscopic theory of preformed FE order parameter is derived by resumming locally the $q \rightarrow 0$ vibrations to obtain a vector field of displacement $\vec{\xi}(x, t)$ which is proportional to an emergent polarization [5]. Upon averaging out the low-energy $\Xi_{q \rightarrow 0}$ (see [17] for details), the effective Hamiltonian for the $T>\omega_{T O}(\Gamma)\left(\equiv \bar{\omega}_{0}\right)$ regime reads

$$
\begin{aligned}
H= & \sum_{k} V_{F} k c_{k}^{\dagger} c_{k}+\sum_{q} \tilde{\omega}_{r}(q) b_{q}^{\dagger} b_{q} \\
& +\sum_{k, q, k^{\prime}} V_{\mathrm{ee}}(k, q) c_{k^{\prime}}^{\dagger} c_{k^{\prime}-q} c_{k}^{\dagger} c_{k+q} \\
& +\sum_{q} \tilde{g}_{2}\left[\hat{\Xi}_{q} \cdot \tilde{\xi}(t)\right]\left(b_{q}+b_{-q}^{\dagger}\right)\left[K \xi_{c}(t)+M \ddot{\xi}_{c}(t)\right] \\
& +\sum_{k, q} F_{e l-p h}\left[k, q ; \xi_{c}(t)\right]\left[c_{k}^{\dagger} c_{k+q}\left(b_{q}+b_{-q}^{\dagger}\right)\right],
\end{aligned}
$$

where we take linear dispersion for the remaining rapid phonons $\tilde{\omega}_{r}(q)=c_{p h} q$ and the last two terms, with $g_{2} \equiv$ $\left.U_{\text {ah }}\right|_{q_{1} \rightarrow 0}$ and $F_{e l-p h} \sim \xi_{c}$, are emergent due to the spontaneous symmetry breaking described by $\vec{\xi}(x, t)$. Terms with such an operator content have been derived using a different formalism in Ref. [18]. Here we take $\vec{\xi}(x, t)=\xi_{c}(t) \varphi(x) \otimes \tilde{\xi}$ with a spatial profile $\varphi(x)$ [19] known from QCP decay. The time dependence of the amplitude $\xi_{c}(t)$ is determined from an anharmonic equation of motion:

$$
\ddot{\xi}_{c}(t)+\tilde{\omega}_{0} \xi_{c}(t)+g\left[\xi_{c}(t)\right]^{3}=0 .
$$

Here $\tilde{\omega}_{0}=\sqrt{K / M}=\min \left[\tilde{\omega}_{r}(q)\right]$ and $g=\left.U_{\text {ah }}\right|_{x \rightarrow 0}$. The pseudospin variable $\tilde{\xi}(t)$, which captures the two possible directions of FE distortion, obeys the Ising-Kondo Hamiltonian:

$$
H_{\tilde{\xi}}=\sum_{q}^{q h} \tilde{g}_{2} \tilde{\xi}_{z}\left(b_{q}+b_{-q}^{\dagger}\right) .
$$

Both Eqs. (3) and (4) define analytically solvable theories. In the following, the parameters that enter into Eqs. (2)-(4), are taken from Refs. [6,14] to make a link with a real material, $\mathrm{PbTe}$. The mutual motion of electrons and ions induces energy transfer between the two subsystems, a central quantity of 
this study. To capture it we need to go beyond the adiabatic approximation. We then take a nonadiabatic case $V_{F}=c_{p h}$, which supports a maximal drag through vertical electron scatterings, i.e., electrons exchange energy with phonons and still remain available for further scattering events along the "heaviest" axis of the Fermi pocket instead of spreading evenly over the entire Fermi surface. The ability to exchange energy with phonons many times maximizes the distortion of occupancies along $\hat{x}$ (see Fig. 1 inset).

The effective model given by Eqs. (2) and (3) reduces our problem to electrons and phonons interacting with a classical variable whose equation of motion is known. To solve the electron-phonon problem we use a variant of correlated electron-ion dynamics, where the analytic equations of motion for electron and phonon densities are integrated. The set includes the momentums' correlation, $\hat{\lambda}(q, t)$ in [20], which is forced to be decoupled in the adiabatic approximation [18], while in our time evolution it follows an unbiased trajectory caused by $F_{e l-p h}$. The simpler, real-space variant of the method, called ECEID [21], was proven to be able to capture nonadiabatic effects beyond Ehrenfest dynamics [22]. In the equation of motion (EOM) for the new $\hat{\lambda}(q, t)$ the two-body density matrix for electrons enters. It is here where the $V_{\text {ee }}$ term intervenes and we tackle it in the random phase approximation generalized to add the susceptibility of a dipole gas to the Lindhard susceptibility and to include the SingwiSjölander local field corrections. We develop our results in two steps: first we compute the effective electron-phonon coupling and then we use it to estimate the transport coefficient.

Electron-TO phonon coupling. The initial state is the thermal equilibrium state $|\Psi\rangle_{T}$ with one phonon added (in a given $q$ mode) [23]: $|\Psi\rangle_{i}=b_{q}^{\dagger}\left(t=t_{0}\right)|\Psi\rangle_{T}$. We study the expectation values of equal-time fermionic occupancies $\left\langle c^{\dagger}(t, k) c(t, k)\right\rangle$ and by monitoring their time dependence, as the extra phonon is propagating through the system, we are able to obtain the transferred energy $\delta E_{k}\left[|\Psi\rangle_{i}\right] \sim \delta n_{k}\left[|\Psi\rangle_{i}\right]$ which is equal to the electron-phonon coupling strength.

The results of the calculation are shown in Fig. 2. Our simulations clearly show the presence of electron-phonon coupling - there is an energy transfer due to an extra phonon into the fermionic system. The electronic densities $\hat{\rho}_{e}$ are not constant; their time evolution is defined by high- and low-frequency oscillations. We are modeling a closed quantum system, so we monitor how the energy is transferred between the available discrete levels. In order to analyze the oscillations we take a Fourier transform of the time dependence. Since the transient state, when phonons are trying to accommodate the extra excitation, is observed only for phonon densities, the spectrum of quasistationary $\hat{\rho}_{e}$ reveals the clear separation of energy scales. The high frequencies can now be associated with the difference of discrete energy levels of the harmonic model (with quantized values of momenta) and are broadened by anharmonicity. At low frequencies we observe the most pronounced peak whose energy scale falls close to $F_{e l-p h}$. We interpret this feature as being present due to Rabi oscillations that develop in our closed quantum system that was initially set out of equilibrium. In this interpretation the excited phonon subsystem exerts its influence on discrete occupations of fermionic levels while the quasiclassical coupling is sufficient to drive the Rabi oscillations. What we
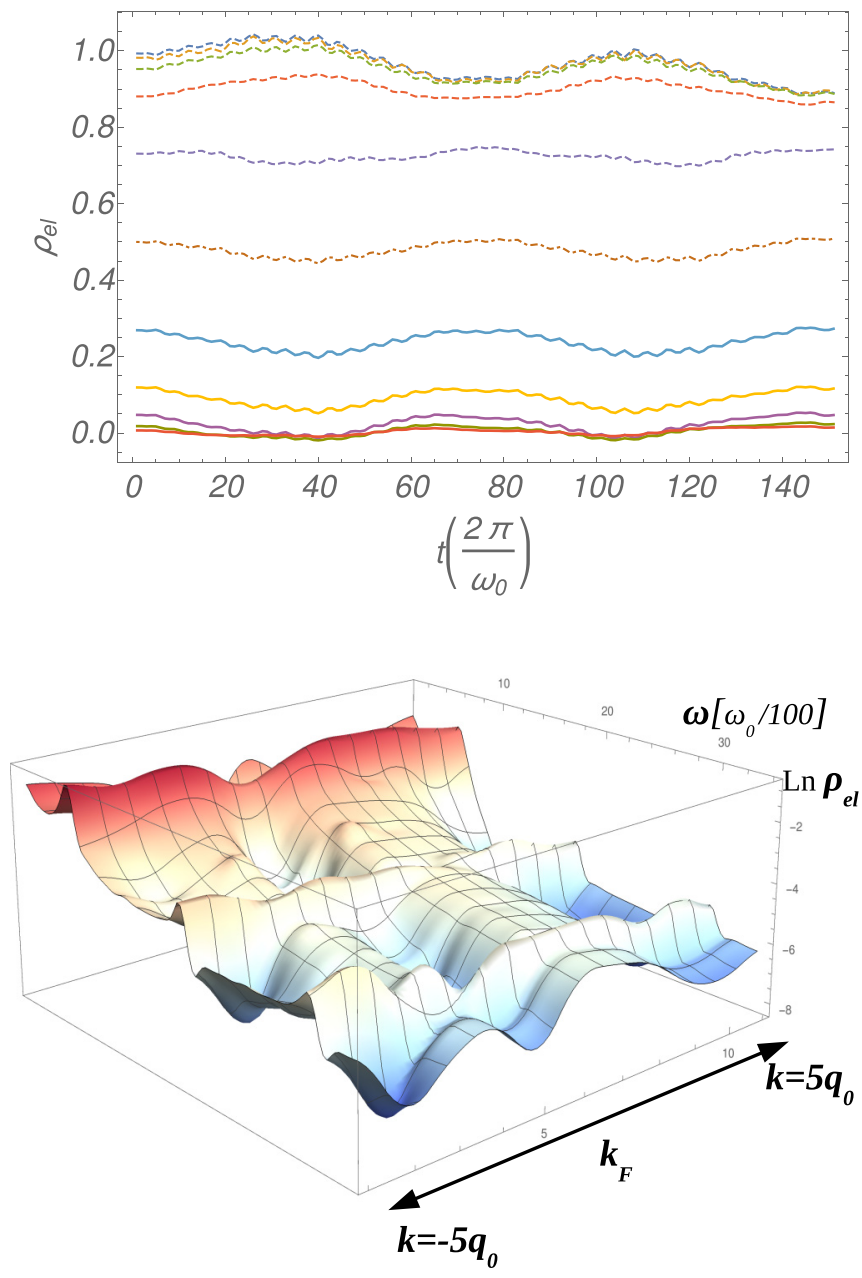

FIG. 2. Response of the fermionic system upon applying a bosonic creation operator in a $q_{1}=1 q_{0}$ mode with energy $1 \tilde{\omega}_{0}$. The calculation is done for discrete bosonic/fermionic modes with energies $E_{n}=n \omega_{0}$ (for fermions $\epsilon=E_{F} \pm E_{n}$ ) where $\omega_{0}$ is the lowest energy of the linear part of the TO spectrum (hence around $20 \%$ above $\left.\bar{\omega}_{0}\right), n \in[1,5]$. In the top panel the temporal evolution of expectation values of fermion occupancies are shown for each bosonic mode; this is a solution of our EOM. In the bottom panel we show its Fourier transform (on a natural logarithmic scale), hence an effective electron-TO phonon coupling as a function of energy and (fermion's) momentum.

observe is in fact a dynamics of respective Floquet states. The varying amplitude of oscillation for various fermionic states informs us about the scattering dependence on fermion momentum.

To explore the quantitative effect of the FE-QCP proximity, we need to find how the parameters in Eq. (3) are changing as the system is moved on the $T-\delta$ plane (here $\delta$ is a control parameter, e.g., strain). The renormalization group flow of scalar $\phi^{4}$ theory [24] is known to give a correct description of the FE-QCP [13,25]; then we know universal flow of the Gruneisen parameter [26] and its relation with the anharmonicity [27].

Transport coefficients. We focus on the Seebeck coefficient which can be expressed in terms of Kubo current-current susceptibilities $L^{i j}: S=L^{\sigma, \kappa} /\left(T L^{\sigma, \sigma}\right)$ where $L^{\sigma, \kappa}$ will contain 


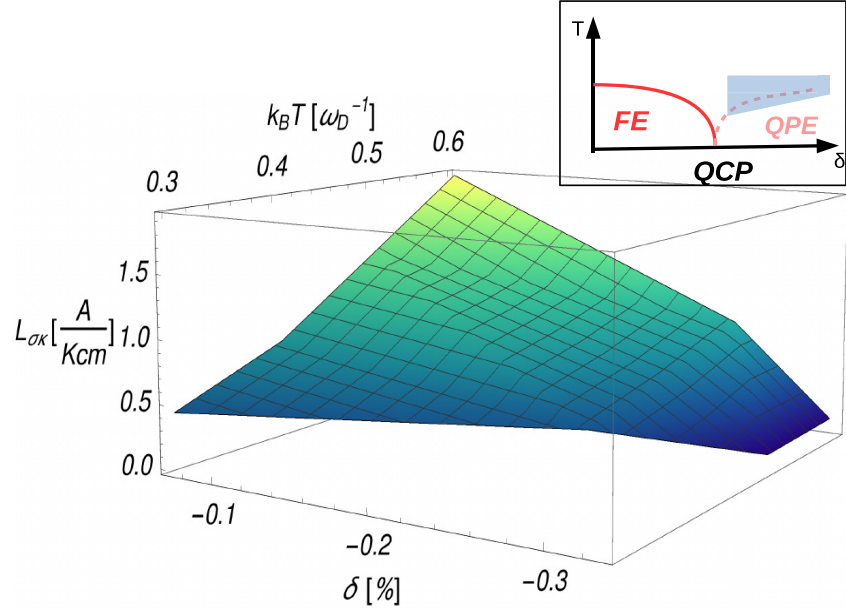

FIG. 3. Thermoelectric conductance $L_{d}^{\sigma, \kappa}$ as the system approaches the FE-QCP, where the QCP's vicinity is parametrized as a function of strain and temperature. The natural unit of thermoelectric conductance is $\hbar e / k_{B}$, the natural length scale is $2 \pi / q_{0}$, and the energy unit throughout the calculation is $\omega_{0}$. By taking $\left.\omega_{0}^{\mathrm{PbTe}}\right|_{\delta=-0.3 \%}=1 \mathrm{THz}$ it allows us to estimate the amplitude of the effect on a vertical scale. The inset shows the region in the parameter space (gray) where our reasoning applies. PbTe would be located on the right side of the gray zone.

both an electronic liquid dissipative part $L_{e l}^{\sigma, \kappa}$ and a new phonon-drag part $L_{d}^{\sigma, \kappa}$. As the system approaches the FE-QCP it is known that the electronic resistivity $\left(\sim 1 / L^{\sigma, \sigma}\right)$ increases but at the same time the Fermi pockets shrink so the entropy associated with these carriers $\left(\sim L_{\mathrm{el}}^{\sigma, \kappa}\right)$ also decreases. We then aim to compute the drag component $L_{d}^{\sigma, \kappa}$ to check if it is able to profit from QCP fluctuations of $\xi_{c}$.

From the time evolution of the densities $\Delta \rho_{e}\left(\epsilon_{i}, t\right)$ one can extract expectation values of electron and phonon currents, and compute cross-correlation functions of currents' variations [28]. Extra care needs to be taken, since the output of our simulation is a superposition of Floquet states. However, one may argue that the drag effect itself takes place in the presence of the unequilibrated state of the phonon distribution $N_{B}^{(0)}+\Delta N_{B}$. Hence our description captures the experimental situation rather well. We then apply the Kubo formalism adapted, by the Lehmann representation of currents $\left\langle j_{\kappa}^{i}\right\rangle$ crosscorrelation functions, for the Floquet states [29]

$$
L^{\sigma, \kappa}=\imath \sum_{i, j, \omega_{n}<T} \frac{n_{F}(i)-n_{F}(j)}{\epsilon_{i}-\epsilon_{j}} \frac{\left\langle\left\langle j_{\sigma}^{i}\right\rangle\right\rangle\left\langle\left\langle j_{\kappa}^{j}\right\rangle\right\rangle}{\epsilon_{i}-\epsilon_{j}+\omega_{n}+\imath \delta^{+}},
$$

where $n_{F}(i)$ is a Fermi-Dirac distribution for a state with energy $\epsilon_{i}$, the summation is over the Floquet states (oscillation index $n$ ), over all available modes $i, j$, and the double average indicates average in time and over all possible initial/final states in an energy window around a given discrete level.

The result of this procedure is shown in Fig. 3. The temporal fluctuations of both densities can produce a net correlation between respective currents, hence the drag effect. The effect becomes more pronounced as the system approaches the QCP, where the relative effect of $\xi_{c}$ fluctuations is enhanced. This should be compared with a maximal $L^{\sigma, \kappa}$ measured in PbTe, $L^{\sigma, \kappa}=0.5 \mathrm{~A} /(\mathrm{K} \mathrm{cm})$, which indicates that the effect proposed here may contribute significantly. Our plot is restricted to a regime where our approximation applies (inset). As we increase temperature, we shall ultimately enter into a regime, not captured in our minimal model, where the thermal fluctuations destroy the temporal coherence of $\xi_{c}$ [30] and furthermore, in the case of $\mathrm{PbTe}$, relaxation into LA modes will appear [16]. Hence the increase of $L^{\sigma, \kappa}$ should be suppressed when $T \gtrsim \omega_{D}$ [31]. For all points on the plot in Fig. 3 we have used the same initial condition $\Delta N_{B}(q)$ proportional to polylogarithmic functions [32] so the observed $T-\delta$ dependence comes from the critical dynamics of the system, not from a biased choice of the initial condition. The changes of $L^{\sigma, \kappa}$ in Fig. 3 are solely due to modified parameters of the EOM, which in turn follow modifications (of, e.g., $g_{2}$ [26]) known from the $\phi^{4}-\mathrm{QCP}$ theory [24]. The increase of thermoelectric conductivity (drag) is exclusively due to increased anharmonicity, as duly captured in our model.

The broad importance of this work stems from our method that goes beyond adiabatic approximation-a paradigm, since Born-Oppenheimer work [33] almost a century ago, that has turned out to be insufficient with the advent of theoretical [29,34] and experimental [35] quantum nonadiabatic and nonequilibrium dynamics. Formally, the adiabatic ansatz means that the Fock spaces for many-body bosonic and fermionic states are decoupled into a simple product, which implies that their entropies are additive. In practice, applying the phonon creation operator (in the adiabatic basis) does not alter the many-body state of fermions. Our formalism then clearly allows one to go beyond this approximation: in Fig. 2 we directly observe a change of electronic density of states $\Delta \hat{\rho}_{e}$ after $b_{q}^{\dagger}\left|\Psi_{G}\right\rangle$. The phonon-induced off-diagonal terms $\left\langle\Psi_{+q}\left|b_{q}^{\dagger}\right| \Psi_{G}\right\rangle=t_{+q-G} \neq 0$ are precisely those neglected in the adiabatic approximation. Our result provides direct access to a measure of nonadiabaticity in the system and furthermore, through $\hat{\rho}_{e}=\hat{\rho}_{e}^{(0)}+\Delta \hat{\rho}_{e}$, all electronic observables can be computed. Here we have chosen to focus on one quantity, the thermoelectric conductivity, which is directly proportional to the entropy transfer between the two subsystems. Furthermore, we choose the simplest model, with a linear coupling to the TO mode (otherwise prohibited in the high-symmetry structure) and all other ingredients (critical dynamics, anharmonic motion) obey exact analytical solutions. This is for the sake of transparency, to study a situation where nonadiabatic phenomena manifest particularly clearly.

In conclusion we have shown that close to a FE-QCP strong anharmonic fluctuations of atomic positions, described by a collective variable $\xi_{c}$, lead to a new type of coupling between electrons and anharmonic phonons. It is a strong-coupling effect that one is able to detect only by going not only beyond the standard adiabatic approximation for electrons but also beyond Ehrenfest dynamics for ionic motion. Our model is applicable in any distortive QCP where electronic polarizabilities are modified. Furthermore we have shown that the coupling through $\xi_{c}$ can produce a finite net phonon drag effect, which substantially enhances thermoelectric transport coefficients. A superconducting phase, in an analog material $\mathrm{SrTiO}_{3}$, is present even closer to the QCP and therein the source of a strong electron-TO phonon coupling remains a mystery. A possible extension of this work is to cover this intriguing regime. 
Acknowledgments. I am greatly indebted to Sucismita C. Chutia and Jorge Kohanoff for a careful reading of the manuscript and many valuable remarks. I also wish to thank T. Todorov and P. Aguado Puente for many helpful discussions.
This work was supported by a research grant from Department for the Economy Northern Ireland and Science Foundation Ireland (SFI) under the SFI-DfE Investigators Programme Partnership, Grant No. 15/IA/3160.
[1] P. Gorai, V. Stevanović, and E. S. Toberer, Computationally guided discovery of thermoelectric materials, Nat. Rev. Mater. 2, 17053 (2017).

[2] J.-H. Pöhls, Z. Luo, U. Aydemir, J.-P. Sun, S. Hao, J. He, I. G. Hill, G. Hautier, A. Jain, X. Zeng, C. Wolverton, G. Jeffrey Snyder, H. Zhu, and M. A. White, First-principles calculations and experimental studies of $X Y Z_{2}$ thermoelectric compounds: Detailed analysis of van der Waals interactions, J. Mater. Chem. A 6, 19502 (2018).

[3] Z. M. Gibbs, F. Ricci, G. Li, H. Zhu, K. Persson, G. Ceder, G. Hautier, A. Jain, and G. J. Snyder, Effective mass and Fermi surface complexity factor from ab initio band structure calculations, npj Comput. Mater. 3, 8 (2017).

[4] Y. Pei, H. Wang, and G. J. Snyder, Band engineering of thermoelectric materials, Adv. Mater. 24, 6125 (2012).

[5] A. F. Devonshire, XCVI. Theory of barium titanate, Philos. Mag. 40, 1040 (1949).

[6] Y. Zhang, X. Ke, P. R. C. Kent, J. Yang, and C. Chen, Anomalous Lattice Dynamics Near the Ferroelectric Instability in PbTe, Phys. Rev. Lett. 107, 175503 (2011).

[7] M. P. Jiang, M. Trigo, I. Savić, S. Fahy, É. D. Murray, C. Bray, J. Clark, T. Henighan, M. Kozina, M. Chollet, J. M. Glownia, M. C. Hoffmann, D. Zhu, O. Delaire, A. F. May, B. C. Sales, A. M. Lindenberg, P. Zalden, T. Sato, R. Merlin, and D. A. Reis, The origin of incipient ferroelectricity in lead telluride, Nat. Commun. 7, 12291 (2016).

[8] C. Herring and E. Vogt, Transport and deformation-potential theory for many-valley semiconductors with anisotropic scattering, Phys. Rev. 101, 944 (1956).

[9] Q. Song, T.-H. Liu, J. Zhou, Z. Ding, and G. Chen, Ab initio study of electron mean free paths and thermoelectric properties of lead telluride, Mater. Today Phys. 2, 69 (2017).

[10] J. Cao, J. D. Querales-Flores, A. R. Murphy, S. Fahy, and I. Savić, Dominant electron-phonon scattering mechanisms in n-type PbTe from first principles, Phys. Rev. B 98, 205202 (2018).

[11] Based on a displacement potential scenario which is implicitly equivalent to an advanced version of the above argument.

[12] B. Sangiorgio, E. S. Bozin, C. D. Malliakas, M. Fechner, A. Simonov, M. G. Kanatzidis, S. J. L. Billinge, N. A. Spaldin, and T. Weber, Correlated local dipoles in PbTe, Phys. Rev. Mater. 2, 085402 (2018).

[13] R. Roussev and A. J. Millis, Theory of the quantum paraelectric-ferroelectric transition, Phys. Rev. B 67, 014105 (2003).

[14] A. H. Romero, E. K. U. Gross, M. J. Verstraete, and O. Hellman, Thermal conductivity in PbTe from first principles, Phys. Rev. B 91, 214310 (2015).

[15] See Supplemental Material at http://link.aps.org/supplemental/ 10.1103/PhysRevResearch.2.012048 for the derivation of $\omega_{\text {TO }}$ renormalization.
[16] O. Delaire, J. Ma, K. Marty, A. F. May, M. A. McGuire, M.-H Du, D. J. Singh, A. Podlesnyak, G. Ehlers, M. D. Lumsden, and B. C. Sales, Giant anharmonic phonon scattering in PbTe, Nat. Mater. 10, 614 (2011).

[17] See Supplemental Material at http://link.aps.org/supplemental/ 10.1103/PhysRevResearch.2.012048 for the derivation of each term in equations Eqs. (2)-(4).

[18] A. P. Horsfield, D. R. Bowler, A. J. Fisher, T. N. Todorov, and C. G. Sánchez, Beyond Ehrenfest: Correlated non-adiabatic molecular dynamics, J. Phys.: Condens. Matter 16, 8251 (2004).

[19] Separation of spatial profile relies on the fact that there is an equal number of left/right moving waves that combine into a standing wave.

[20] See Supplemental Material at http://link.aps.org/supplemental/ 10.1103/PhysRevResearch.2.012048 for the full set of ECEID's EOM.

[21] V. Rizzi, A new development: ECEID xp, Real-Time Quantum Dynamics of Electron-Phonon Systems (Springer, Cham, 2018).

[22] V. Rizzi, T. N. Todorov, J. J. Kohanoff, and A. A. Correa, Electron-phonon thermalization in a scalable method for real-time quantum dynamics, Phys. Rev. B 93, 024306 (2016).

[23] We are concerned with finite temperature properties, hence an operator $b_{q}^{\dagger}$ is applied not to a ground state, but to an equilibrium, thermal state at given finite temperature $T$.

[24] J. A. Hertz, Quantum critical phenomena, Phys. Rev. B 14, 1165 (1976).

[25] L. J. Spalek, R. P. Smith, M. P. M. Dean, M. Itoh, J. F. Scott, G. G. Lonzarich, S. S. Saxena, and S. E. Rowley, Ferroelectric quantum criticality, Nat. Phys. 10, 367 (2014).

[26] L. Zhu, M. Garst, A. Rosch, and Q. Si, Universally Diverging Grüneisen Parameter and the Magnetocaloric effect Close to Quantum Critical Points, Phys. Rev. Lett. 91, 066404 (2003).

[27] C. H. Lee and C. K. Gan, Anharmonic interatomic force constants and thermal conductivity from Grüneisen parameters: An application to graphene, Phys. Rev. B 96, 035105 (2017).

[28] Strictly speaking, one could also recursively repeat the procedure to compute second-, third-, etc., order approximations for a displaced phonon distribution. Here we assume that linear response holds provided our initial first-order guess $\Delta N_{b}(T)$ is close enough to the real displacement.

[29] T. Oka and H. Aoki, Photovoltaic Hall effect in graphene, Phys. Rev. B 79, 081406 (2009).

[30] L. Pálová, P. Chandra, and P. Coleman, Quantum critical paraelectrics and the Casimir effect in time, Phys. Rev. B 79, 075101 (2009).

[31] $\omega_{D}$ is the Debye frequency which in the case of PbTe is six to eight times larger than $\omega_{0}$. 
[32] See Supplemental Material at http://link.aps.org/supplemental/ 10.1103/PhysRevResearch.2.012048 for the derivation of the distorted distribution $\Delta N_{B}(q)$.

[33] M. Born and R. Oppenheimer, Zur quantentheorie der molekeln, Ann. Phys. (Berlin, Ger.) 389, 457 (1927).
[34] F. Giustino, Electron-phonon interactions from first principles, Rev. Mod. Phys. 89, 015003 (2017).

[35] S. Pisana, M. Lazzeri, C. Casiraghi, K. S. Novoselov, A. K. Geim, A. C. Ferrari, and F. Mauri, Breakdown of the adiabatic Born-Oppenheimer approximation in graphene, Nat. Mater. 6, 198 (2007). 